\title{
Fish Balistes capriscus skin extract-induced relaxation in mesenteric arterial bed of rat
}

\author{
L.S. Cavalli ${ }^{\text {a,b }}$, P.L. Possette ${ }^{\text {a }}$, B. Schmidt ${ }^{\text {a }}$, C. Kruel $^{\text {a }}$, M. Grando ${ }^{\text {a }}$, E. Badiale Furlong ${ }^{\text {, }}$, \\ M.R. Cezar-Vaz ${ }^{\mathrm{d}, \mathrm{e}}$, D.M. Barros ${ }^{\mathrm{a}}$, A.L. Muccillo-Baisch ${ }^{\mathrm{a}, \mathrm{b}, \mathrm{e}, *}$ \\ a Departamento de Ciências Fisiológicas, Universidade Federal do Rio Grande, Rio Grande, Rua Eng. Alfredo Huch 475, Rio Grande, 96201-900, Brazil \\ ${ }^{\mathrm{b}}$ Programa de Pós-Graduação em Fisiologia Animal Comparada, Setor de Farmacologia, Universidade Federal do Rio Grande, \\ Rio Grande, Rua Eng. Alfredo Huch 475, Rio Grande, 96201-900, Brazil \\ ${ }^{\mathrm{c}}$ Departamento de Química, Universidade Federal do Rio Grande, Rio Grande, Rua Eng. Alfredo Huch 475, Rio Grande, 96201-900, Brazil \\ d Departamento de Enfermagem, Universidade Federal do Rio Grande, Rio Grande, Rua Eng. Alfredo Huch 475, Rio Grande, 96201-900, Brazil \\ e Programa de Pós-Graduação em Enfermagem Fundação, Universidade Federal do Rio Grande, Rio Grande, Rua Eng. \\ Alfredo Huch 475, Rio Grande, 96201-900, Brazil
}

Received 1 July 2002; received in revised form 15 May 2003; accepted 15 June 2003

\begin{abstract}
The vasorelaxing activity of the aqueous extract of fish Balistes capriscus skin (AEBc) on mesenteric arterial bed (MAB) of rats was studied. The bolus injections of AEBc (bolus of 5.1, 10.2, 20.5, and $41.1 \mathrm{mg}$ ) significantly inhibited, in a concentration-dependent manner, the maximal contractile response induced by methoxamine $(30 \mu \mathrm{M})$ in $\mathrm{MAB}$. The vasodilatation action of $\mathrm{AE} B c$ is not mediated through $\beta$-adrenoceptors or cyclo-oxigenase, since it was not affected by propranolol $(20 \mu \mathrm{M})$ or diclofenac sodium $(3 \mu \mathrm{M})$. The vasodilator response induced by subsequent addition of AEBc Balistes capriscus in bolus was significantly reduced in water infusion for endothelium removal. Treatment with an inhibitor of NO synthase (L-NAME, $10 \mu \mathrm{M}$ ) decreased AEBc effect. The guanylate cyclase inhibitor methylene blue (MB, $100 \mu \mathrm{M}$ ) had no significant effect on $\mathrm{AEB} B$-induced vasodilatation. These results suggest that the vasorelaxing effect of $\mathrm{AE} B c$ is mediated by endothelium-dependent (NO/EDRF) and endothelium-independent neurally induced vasorelaxation from nonadrenergic and noncholinergic nerves (NO).
\end{abstract}

(C) 2003 Elsevier Ireland Ltd. All rights reserved.

Keywords: Nonadrenergic; Mesenteric arterial bed; Vasodilatation

\section{Introduction}

Investigation of biological, chemical, and ecological phenomena in the marine world contributes to a better understanding of marine habitats, and benefits the applied and basis research in pharmaceutical marine natural products (Konig and Wright, 1995), while research into the pharmacological properties of marine natural products may lead to promising candidates for new drugs and potentially active agents for clinical application. Several marine natural products are, in fact, currently in pre-clinical and clinical evaluation, while others show promising biological activities in in vivo and in vitro assays.

The sesterpenoide manoalide, for example, isolated from the sponge Luffariella variabilis in a program to search for

\footnotetext{
${ }^{*}$ Corresponding author. Tel.: $+55-53-2338656$; fax: $+55-53-2338680$.

E-mail address: abaisch@octopus.furg.br (A.L. Muccillo-Baisch).
}

new anti-inflammatory compounds, proved to be a potent inhibitor of phospholipase C, (Konig and Wright, 1995).

In the city of Rio Grande, Southern of Brazil, the fish Balistes capriscus (tetradontiform, balistidae) has been used by fishermen in the treatment of respiratory disorders, such as asthma and bronchitis as water infusion of dried and powdered skin.

The present study was carried out to investigate the effects of aqueous extract of Balistes capriscus skin on isolated mesenteric arterial bed (MAB) precontracted with methoxamine for possible vascular relaxing activity.

\section{Material and methods}

\subsection{Biological materials}

Samples of the fish Balistes capriscus were collected during 1999 and 2000 along the coast of Rio Grande, RS 
(southern Brazil) and identified by Dr. Manoel Haimovich from the Department of Oceanography of Fundação, Universidade Federal do Rio Grande (FURG), Rio Grande, Brazil. A voucher specimen has been deposited at the FURG fish collection (acquisition numbers 83.0245 and 83.0295). The animals were frozen at $-18{ }^{\circ} \mathrm{C}$ until used for the assays.

\subsection{Extract}

Fish skins were dried/toasted in a conventional oven, at the temperature of approximately $180^{\circ} \mathrm{C}$. Coarsely powdered dried skin material $(35 \mathrm{~g})$ was extracted with $120 \mathrm{ml}$ of bi-distilled water using a Soxhlet extractor, yielding, after lyophilization, $4.79 \mathrm{~g}$ of powder $(13.7 \%)$. The extract was stored and dissolved in Krebs Ringer bicarbonate immediately before all tests were performed. All results are shown as milligram of lyophilized powder.

\subsection{Animals}

Male Wistar rats, weighing $419.88 \pm 6.25 \mathrm{~g}(n=52)$, were obtained from Animal House of the Fundação Universidade Federal do Rio Grande. The rats were housed in temperature-controlled rooms $\left(20-22^{\circ} \mathrm{C}\right)$, with a 12-h-light/12-h-dark photoperiod and $55 \pm 1 \%$ relative humidity. Standard laboratory chow (Nuvital, Nuvital Nutrientes, Colombo, Paraná, Brazil) and drinking water were provided ad libitum.

\subsection{Perfused mesenteric arterial vascular bed of the rat (MAB)}

The MAB was removed from animals under brief ether anesthesia and perfused as described by Mc Gregor (1965), as modified by Silva et al. (1984). The superior mesenteric artery with its nerve plexus was carefully separated from the surrounding tissues at a point $2 \mathrm{~cm}$ distal to the aorta. A stainless steel cannula was inserted into the artery. The associated vascular bed was covered with cotton cloth moistened with Krebs Ringer bicarbonate and perfused by means of a peristaltic pump (Milan, Colombo, Brasil) at $5 \mathrm{ml} / \mathrm{min}$ with a similar solution. The composition of the perfusion solution was $(\mathrm{mM}): \mathrm{NaCl}, 118 ; \mathrm{KCl}, 4.7$; $\mathrm{CaCl}_{2}, 3.3 ; \mathrm{KH}_{2} \mathrm{PO}_{4}, 1.2 ; \mathrm{MgSO}_{4}, 2.4 ; \mathrm{NaHCO}_{3}, 25$; glucose, 10; EDTA, 0.03; ascorbic acid, 0.1. The Krebs Ringer bicarbonate solution was gassed with $95 \% \mathrm{O}_{2}$ and $5 \% \mathrm{CO}_{2}$ to obtain a $\mathrm{pH}$ of $7.2-7.4$ and was maintained at $37^{\circ} \mathrm{C}$.

Perfusion pressure was measured with a transducer (Hewlett-Packard, USA) on a side arm, just before the perfusing cannula, and was continuously recorded on a polygraph (Hewlett-Packard). Since the flow rate was constant throughout the whole experimental period, any pressure alteration reflected changes in vascular resistance. All preparations were allowed to equilibrate for at least $30 \mathrm{~min}$ before the start of the experiments.

\subsection{Vasodilatory responses}

In order to evaluate the vasoactive effect of the extracts and perivascular nerve stimulation (PNS), the perfusion pressure of MAB was increased by infusing methoxamine $(30 \mu \mathrm{M})$. When a plateau was reached, the extract and all agonists were administered by bolus injection $(50 \mu \mathrm{M})$. Drugs and extract-induced relaxation $(5.1,10.2,20.5$, and $41.1 \mathrm{mg}$ ) was expressed as a percentage of the pressure induced by methoxamine $(30 \mu \mathrm{M})$.

In experiments with PNS two electrodes, one placed around the hypodermic needle used to cannulate the superior mesenteric artery, the other resting on the vasculature in a lower part of the vascular bed, were used to create transmural field stimulation (amplitude supra maximal of $60 \mathrm{~V}$ and pulse duration of $0.5 \mathrm{~ms}$ ). Currents were applied at various frequencies and time using an electronic stimulator (HSE-D 7801-Hugstetten, Germany).

In order to block the vasoconstrictive action of perivascular sympathetic innervation, the MAB was perfused continuously with guanetidine $(5 \mu \mathrm{M})$, introduced after a period of equilibration. To evaluate vasodilating response, the tone of mesenteric vascular bed was raised by continuous perfusion with methoxamine $(30 \mu \mathrm{M})$, which was added to the perfusate, in order to maintain active tone of the mesenteric vasculature. The solution also contained guanethidine $(5 \mu \mathrm{M})$ to block norepinephrine release. The increased perfusion pressure was allowed to stabilize and the preparation was again subjected to PNS, applied for $30 \mathrm{~s}$.

To study the influence of sensory neurones in the vasodilating responses to the extract or PNS, the preparations were perfused intra- and extra-luminally with capsaicin solution $(0.1 \mu \mathrm{M})$ for $20 \mathrm{~min}$.

\subsection{Drugs}

The following drugs were used: acetylcholine chloride, $N^{\mathrm{G}}$-nitro-L-arginine methyl ester (L-NAME) hydrochloride, methoxamine, papaverine, propranolol, salbutamol, guanethidine, capsaicin, and atropine from Sigma Chemical Co. (St. Louis, MO, USA), while sodium diclofenac (Voltaren ${ }^{\circledR}$ injection solution) was supplied by Novartis (São Paulo, Brazil). The stock solution of capsaicin was prepared in $70 \%$ ethanol; all other compounds were dissolved in distilled water.

\subsection{Statistical analysis}

Data are reported as mean \pm standard error of mean (S.E.M.). Statistical differences between means were evaluated using Student's $t$ test for unpaired and paired observations. The level of significance was $95 \%$. 


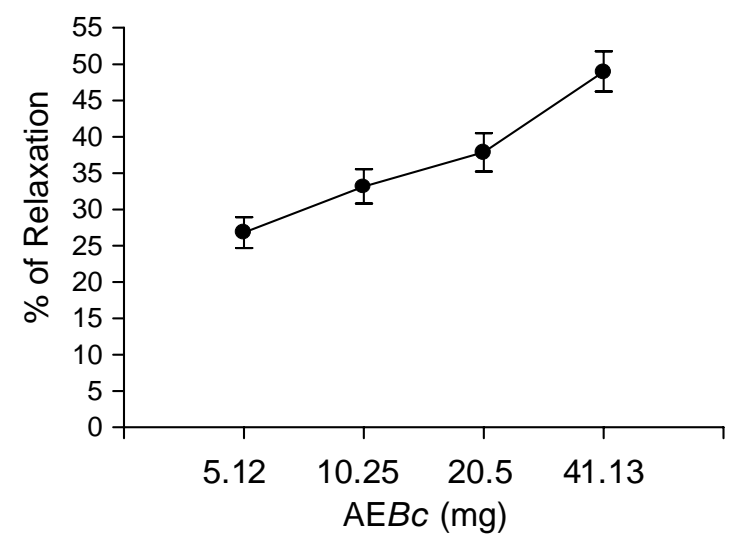

Fig. 1. Dose-response curve for the relaxation induced by different boluses of Balistes capriscus skin aqueous extract $(\mathrm{AEBC})$ in the methoxamine-precontracted mesenteric arterial bed. Each data point on the graph represents the means \pm S.E.M. of 52 experiments.

\section{Results}

\subsection{Vasodilatation in response to aqueous extract of Balistes capriscus skin $(A E B C)$ in MAB precontracted with methoxamine}

The basal perfusion pressure, of the MAB precontracted with methoxamine, was $19.7 \pm 0.7 \mathrm{~mm} \mathrm{Hg}(n=52)$.

$\mathrm{AEBc}(n=52)$ bolus injections $(5.1,10.2,20.5$, and $41.1 \mathrm{mg}$ ) significantly inhibited, in a concentration-dependent manner, the maximal contractile response induced by methoxamine (Fig. 1).

\subsection{Effect of cyclooxygenase pathway inhibitor sodium} diclofenac on changes in vasodilator responses to $A E B c$ in mesenteric arterial bed precontracted by methoxamine

To exclude the involvement of prostaglandins in the extract-induced vasodilatation, the MAB was perfused with

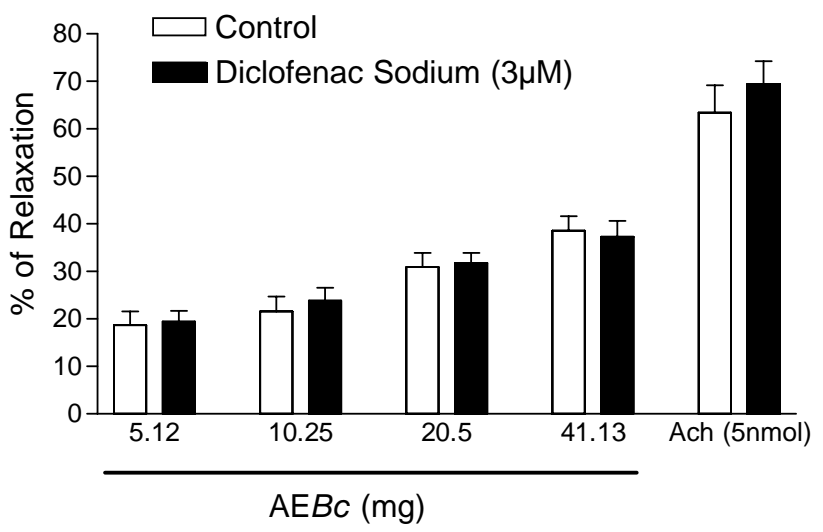

Fig. 2. Effect of sodium diclofenac $(3 \mu \mathrm{M})$ on relaxation response to Balistes capriscus skin aqueous extract (AEBc) and acetylcholine (Ach) in methoxamine-precontracted mesenteric arterial bed. Relaxation is expressed as a percentage of the contraction induced by methoxamine. The values are means \pm S.E.M. $(n=8)$.

diclofenac sodium $(3 \mu \mathrm{M})$ for $30 \mathrm{~min}$. The treatment of preparations with the cyclooxygenase inhibitor $(n=8)$ did not modify $\mathrm{AE} B c$ responses (Fig. 2).

\subsection{Effect of $\beta$-adrenergic pathway inhibitor on $A E B c$-induced vasodilatation in mesenteric arterial bed precontracted with methoxamine}

To exclude the involvement of the $\beta$-adrenergic pathway on extract-induced vasodilatation, the $\operatorname{MAB}(n=6)$ was perfused with propranolol $(20 \mu \mathrm{M})$ for $30 \mathrm{~min}$. The treatment of preparations with the inhibitor did not modify AEBC response (Fig. 3). On the other hand, the vasodilatory effect of salbutamol $(8.0 \mathrm{nmol})$, a $\beta$-adrenergic agonist, was significantly modified, from $32.59 \pm 3.85 \%$ to $14.33 \pm 4.97 \%$ $(n=6)$ in the presence and absence of propranolol, respectively.

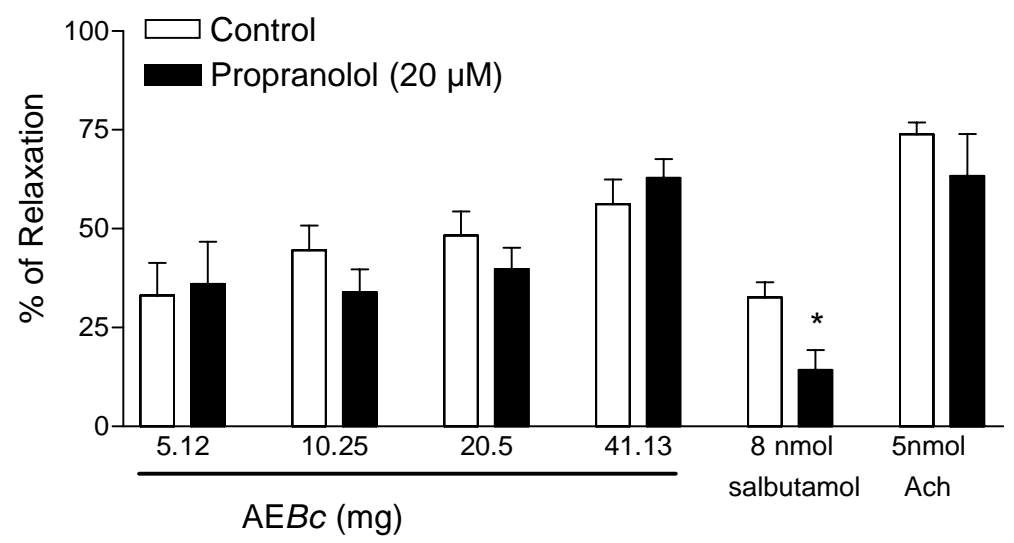

Fig. 3. Effects of propanolol $(20 \mu \mathrm{M})$ on relaxation response to Balistes capriscus aqueous extract (AEBc), salbutamol and acetylcholine (Ach) in methoxamine-precontracted mesenteric arterial bed. Relaxation is expressed as a percentage of the contraction induced by methoxamine. The values are means \pm S.E.M. $(n=6)$. $(*)$ Denotes value significantly different $(P<0.05)$ from the control. 


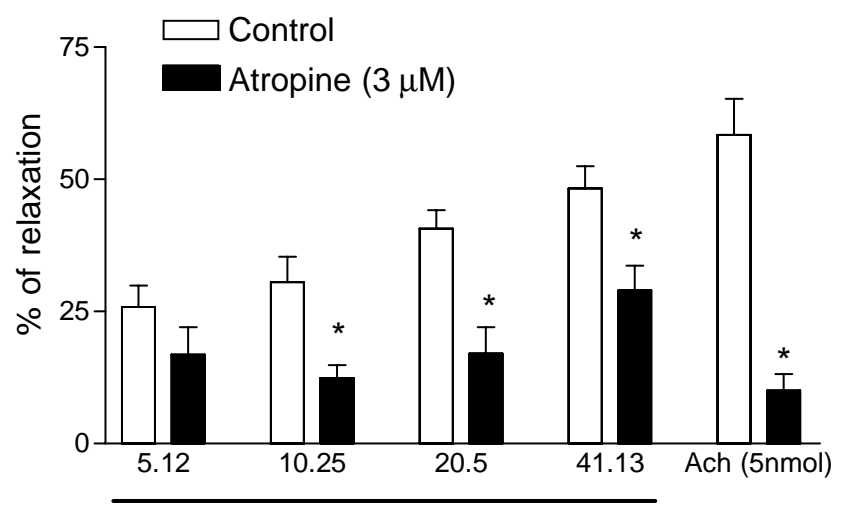

$\mathrm{AEBc}(\mathrm{mg})$

Fig. 4. Effects of atropine $(3 \mu \mathrm{M})$ on relaxation response to Balistes capriscus skin aqueous extract (AEBc) and acetylcholine (Ach) in methoxamine-precontracted mesenteric arterial bed. Relaxation is expressed as a percentage of the contraction induced by methoxamine. The values are means \pm S.E.M. $(n=7)$. $(*)$ Denotes value significantly different $(P<0.05)$ from control.

\subsection{Effect of muscarinic pathway inhibitor on AEBc and} acetylcholine-induced vasodilatation in mesenteric arterial bed precontracted with methoxamine

To study the involvement of muscarinic pathway on extract-induced vasodilatation, the MAB was perfused with atropine for $30 \mathrm{~min}$. As shown in Fig. 4, the extract-induced vasodilatation of the MAB were significantly inhibited $(P<0.05)$ by treatment with atropine $(3 \mu \mathrm{M})$. The same procedure decreased the acetylcholine-induced vasodilatation from $58.38 \pm 6.82 \%$ to $10.0 \pm 3.1 \%$.

\subsection{Effect of capsaicin treatment on changes in perfusion} pressure induced by $A E B C$ and perivascular nerve stimulation in MAB precontracted with methoxamine

Capsaicin $(0.1 \mu \mathrm{M})$ pretreatment for 20 min prevented the vasodilatation induced by aqueous extract of Balistes

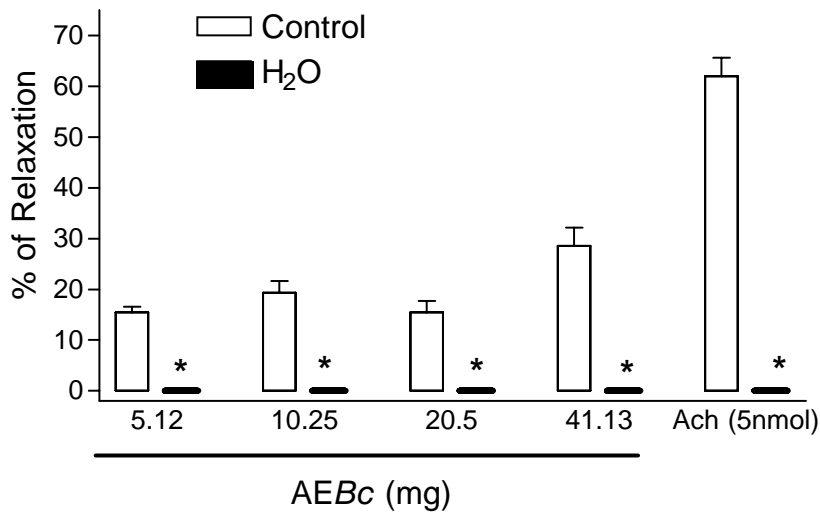

Fig. 6. Effects of endothelium removal on relaxation response to Balistes capriscus skin aqueous extract $(\mathrm{AE} B c)$ and acetylcholine (Ach) in methoxamine-precontracted mesenteric arterial bed. Relaxation is expressed as a percentage of the contraction induced by methoxamine. The values are means \pm S.E.M. $(n=8)$. $(*)$ Denotes value significantly different $(P<0.05)$ from control.

capriscus, but only for the bolus of $41.3 \mathrm{mg}$, from $63.97 \pm$ $6.15 \%$ to $44.19 \pm 7.0 \%$ (Fig. 5).

The response of MAB to PNS in the presence of capsaicin was inhibited from $22.28 \pm 2.66 \%$ to $8.78 \pm 2.97 \%$ (Fig. 5).

3.6. Effect of endothelium removal on changes in perfusion pressure induced by $A E B c$ and acetylcholine in $M A B$ precontracted with methoxamine

To exclude the involvement of endothelium on extractinduced vasodilatation, the MAB was perfused with distilled water for $10 \mathrm{~min}$ for endothelium removal (Criscione et al., 1984). In the intact MAB $(n=8)$, contracted with methoxamine $(30 \mu \mathrm{M})$, aqueous extractable fraction caused immediate relaxation. After the endothelium was removed the $\mathrm{AEB} C$-induced vasodilatation was significantly changed (Fig. 6). The same procedure decreased the acetylcholine-induced vasodilatation ( $5 \mathrm{nmol}$, bolus) from $62.0 \pm 3.65 \%$ to zero.

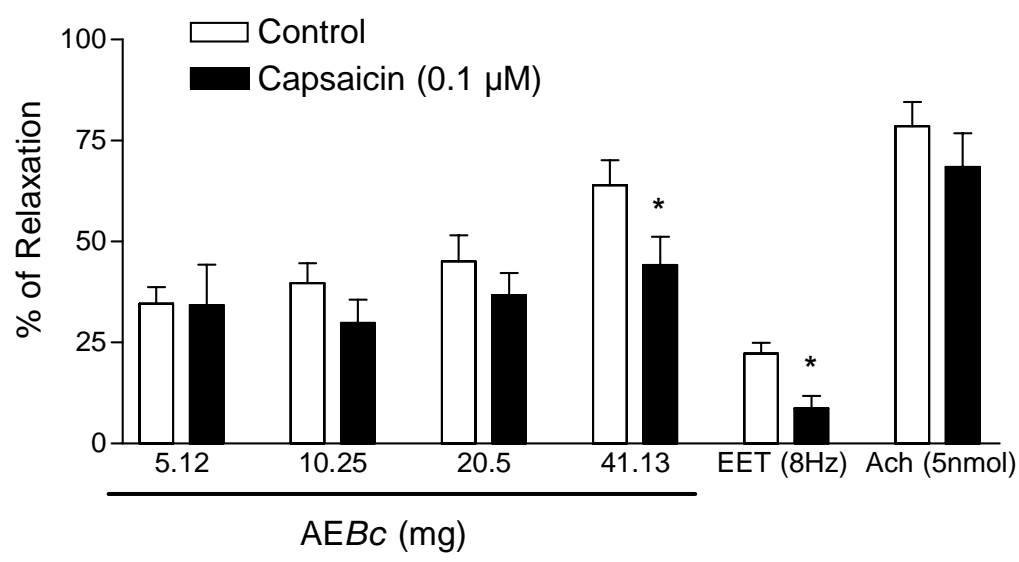

Fig. 5. Effects of capsaicin $(0.1 \mu \mathrm{M})$ on relaxation response to Balistes capriscus skin aqueous extract (AEBc) and PNS-induced vasodilatation in methoxamine-precontracted mesenteric arterial bed. Relaxation is expressed as a percentage of the contraction induced by methoxamine. The values are means \pm S.E.M. $(n=8)$. $(*)$ Denotes value significantly different $(P<0.05)$ from control. 


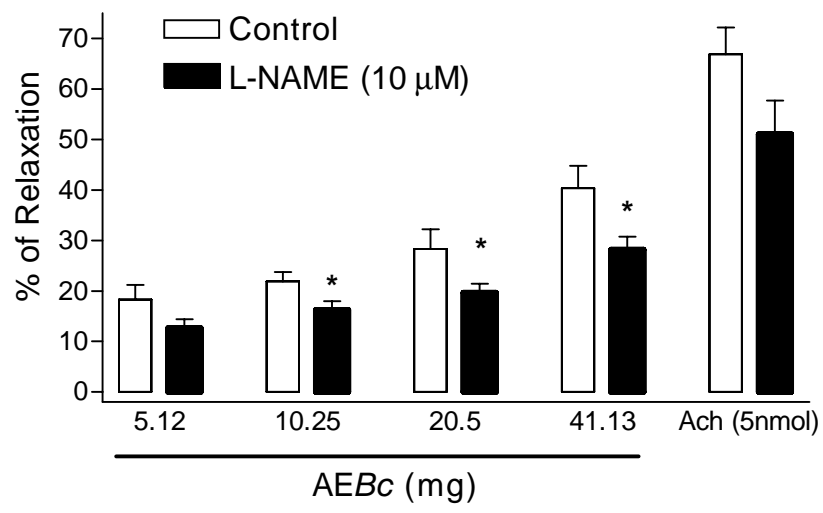

Fig. 7. Effects of $N^{\mathrm{G}}$-nitro-L-arginine methyl ester (L-NAME, $10 \mu \mathrm{M}$ ) on relaxation response to Balistes capriscus skin aqueous extract (AEBc) and acetylcholine (Ach) in methoxamine-precontracted mesenteric arterial bed. Relaxation is expressed as a percentage of the contraction induced by methoxamine. The values are means \pm S.E.M. $(n=8)$. $(*)$ Denotes value significantly different $(P<0.05)$ from control.

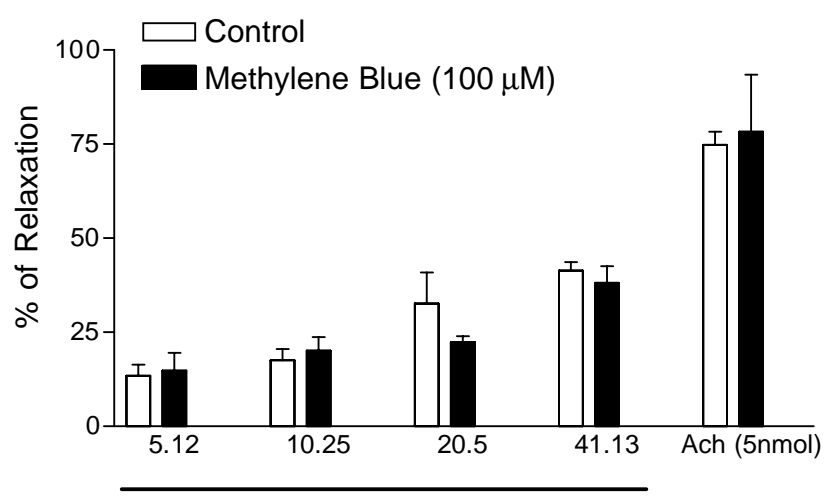

$\mathrm{AEBc}(\mathrm{mg})$

Fig. 8. Effects of methylene blue $(100 \mu \mathrm{M})$ on relaxation response to Balistes capriscus skin aqueous extract (AEBc), and acetylcholine (Ach) in methoxamine-precontracted mesenteric arterial bed. Relaxation is expressed as a percentage of the contraction induced by methoxamine. The values are means \pm S.E.M. $(n=7)$.

\subsection{Effect of endothelium-derived NO-cyclic GMP} pathway on vasodilating responses to $A E B c$ in mesenteric arterial bed-precontracted with methoxamine

As shown in Fig. 7, the treatment of preparation with an inhibitor of NO synthase L-NAME $(10 \mu \mathrm{M})$ significantly reduced the $\mathrm{AE} B c$-induced vasodilatation.

The effect of guanylate cyclase inhibitor methylene blue $(\mathrm{MB}, 100 \mu \mathrm{M})$ on the vasodilatation induced by $\mathrm{AEBC}$ was also examined. The treatment of preparations with the inhibitor did not significantly modify the $\mathrm{AE} B C$ response (Fig. 8).

\section{Discussion}

The results of the present study demonstrate, for the first time, that the skin of Balistes capriscus, used in popular medicine in Southern Brazil for treatment of respiratory disorders, produced a dose-dependent and reversible inhibition of methoxamine-induced vascular tone.

The vasorelaxant action of the extract does not appear to involve activation of $\beta$-adrenoceptors, since incubation of the preparations with propranolol failed to affect its vasorelaxation action.

The relaxation induced by the extract was not affected by sodium diclofenac, which indicates that it is not indirectly mediated by the release of prostanoid metabolite(s) derived from the cyclooxygenase pathway of arachidonic acid.

As is well known, the vasorelaxing action of acetylcholine requires the presence of an intact endothelium. The binding of acetylcholine to muscarinic receptors on endothelial cells trigger the release of a potent vasodilator called endothelium-derived relaxing factor (EDRF), discovered by Furchgott and Zawadzki (1980). The EDRF, as nitric oxide (NO), is synthesized from guanidino groups of L-arginine (Palmer et al., 1987) and has been reported to stimulate the production of cGMP, which induces a vasorelaxation in smooth muscle cells (Winquist et al., 1984).

The treatment of the preparations with atropine, at concentration where Ach-mediated vasorelaxation was antagonized (Muccillo Baisch et al., 1998), prevents a great part of the vasorelaxant action of the extract of Balistes capriscus skin in MAB. In addition, when the MAB was perfused with distilled water, the vasorelaxant actions of acetylcholine and of the extract were greatly reduced. As exposure to distilled water destroys the endothelial cells (Criscione et al., 1984), these results confirm that in MAB of rats, both acetylcholine (Muccillo Baisch et al., 1998) and the extract-induced vasodilatation are mediated by the release of endothelium-derived substances. Taken together, such results suggest that the vasorelaxant actions of the extract are mediated jointly by endothelium-dependent cholinergic mechanism.

The vasorelaxant effects of endothelium-dependent substances could be inhibited by several L-arginine analogues such as $N$-monomethyl-L-arginine (L-NMMA) and $N^{\mathrm{G}}$-nitro-L-arginine methyl ester (L-NAME) (Palmer et al., 1987; Rees et al., 1989 and Moore et al., 1990). In the present study the aqueous extract of Balistes capriscus dried skin caused endothelium-dependent relaxation, which was reversed by L-NAME, at a concentration which Ach-mediated vasorelaxation was antagonized. This suggests that certain compounds present in the skin extract may cause relaxation by increasing $\mathrm{NO}$ or other related compounds.

The endothelium-dependent vasorelaxants, such as acetylcholine, could also release endothelium-derived hyperpolarizing factor (EDHF) from the endothelium, which induces vasorelaxation through membrane hyperpolarization (Adeagbo and Triggle, 1993). The possible existence of a novel endothelium-derived relaxing factor in the endothelium of rat MAB was examined in the study of Kamata et al. (1996). The authors suggested that one or more EDRF must exist, other than NO or EDHF. The novel EDRF may relax 
the MAB through production of cAMP, but not cGMP. This suggestion could support the finding that the vasorelaxant effect induced by $\mathrm{AEB} C$ was not inhibited by methylene blue. However, methylene blue is not a potent guanylate cyclase inhibitor, and has also been shown to inhibit NO's activity (Mayer et al., 1993; Lou et al., 1995).

In the MAB, the relaxation induced by PNS has been determined to be mediated by the activation of capsaicinsensitive sensory fibers which releases neuropeptids locally, with a potent vasorelaxation action on intestinal blood flow (Manzini and Perreti, 1988). Thus, the present investigation has demonstrated the presence of a nonadrenergic, noncholinergic vasodilator innervation in rat mesenteric arteries.

In the present study, the vasodilatation induced by aqueous extract from Balistes capriscus skin was reduced in the presence of capsaicin, a pungent ingredient in chili pepper (Capsicum sp.), which has been used to damage selectively the subpopulation of sensory neurons and to deplete neurotransmitter, such as substance P and CGRP (Holzer, 1988). This suggests that the Balistes capriscus skin-induced MAB vasodilatation is mediated by primary sensory nerve fibers. Consequently these data show that in the MAB, the vasodilatation induced by the aqueous extract of Balistes capriscus skin is mediated by endothelium-dependent mechanism mediated by EDHF or NO and endothelium-independent neurally induced relaxation, associated with NO from a nonadrenergic noncholinergic nerves.

Pharmacological and chemical studies are currently in progress to isolate and characterize the active compounds present in the fish skin, and also to investigate the possible mechanism of action.

\section{Acknowledgements}

The authors are grateful to Prof. Dr. Manoel Haimovich, for the donation and identification of biological material, Mr. Robaldo, M.Sc. for help in the taxonomic identification of the fish, Walquíria Lopes for technical assistance, Conselho Nacional de Desenvolvimento Científico e Tecnológico $(\mathrm{CNPq})$ for Mayeve Grando and Carla Kruel financial assistance, Coordenação de Aperfeiçoamento de Pessoal de Nível Superior (CAPES) for M.Sc. fellowship to Lisandra Cavalli, and Fundação de Amparo a Pesquisa do Rio Grande do Sul (FAPERGS) for Paula Lidiane Possette's financial assistance. Thanks are also due to Dr. Euclydes dos Santos $\mathrm{F}^{\mathrm{o}}$ and Dr. Luiz Eduardo Maia Nery for assisting in the revision of the manuscript.

\section{References}

Adeagbo, A.S.O., Triggle, C.R., 1993. Varying extracellular $\left[\mathrm{K}^{+}\right]$: a functional approach to separating EDHF- and EDNO-related mechanisms in perfused rat mesenteric arterial bed. Journal of Cardiovascular Pharmacology 21, 423-429.

Criscione, L., Müller, K., Prescott, M.F., 1984. Endothelial cells loss enhances the pressor response in resistance vessels. Journal of Hypertension 2, 441-444.

Furchgott, R.F., Zawadzki, J.V., 1980. The obligatory role of endothelial cells in the relaxation of arterial smooth muscle by acetylcholine. Nature 299, 373-376.

Holzer, P., 1988. Local effector functions of capsaicin-sensitive sensory nerve endings: involvement of tachykinins CGRP and other neuropeptids. Neuroscience 24, 739-768.

Kamata, K., Numazawa, T., Kasuya, Y., 1996. Characteristics of vasodilatation induced by acetylcholine and platelet-activating factors in the rat mesenteric arterial bed. European Journal of Pharmacology 298, 129-136.

Konig, G.M., Wright, A.D., 1995. Marine natural products research: current directions and future potencial. Planta Medica 62, 193-211.

Lou, D., Das, S., Vincent, S.R., 1995. Effects of methylene blue and LY83563 on neuronal nitric oxide synthase and NADPH-diaphorase. European Journal of Pharmacology 290, 247-251.

Manzini, S., Perreti, F., 1988. Vascular effects of capsaicin in isolated perfused rat mesenteric bed. European Journal of Pharmacology 148, 153-159.

Mayer, B., Brunner, F., Schmidt, K., 1993. Inhibition of nitric oxide synthesis by methylene blue. Biochemical Pharmacology 45, 367374.

Mc Gregor, D.D., 1965. The effect of sympathetic nerve stimulation on vasoconstritor responses in perfused mesenteric blood vessel of the rat. Journal of Physiology 177, 21-30.

Moore, P.K., al-Swayeh, O.A., Chong, N.W.S., Evans, R.A., Gibson, A., 1990. L- $N^{\mathrm{G}}$ - nitro arginine (L-NOARG), a novel, L-arginine-reversible inhibitor of endothelium-dependent vasodilatation in vitro. British Journal of Pharmacology 99, 408-412.

Muccillo Baisch, A.L., Johnston, K.B., Paganini Stein, F.L., 1998. Endothelium-dependent vasorelaxing activity of aqueous extract of Ilex paraguariensis on mesenteric arterial bed of rats. Journal of Ethnopharmacology 60, 133-139.

Palmer, R.M.J., Ferridge, A.G., Mocada, S., 1987. Nitric oxide release accounts for the biological activity of endothelium-derived relaxing factor. Nature 327, 524-526.

Rees, D.D., Palmer, R.M.J., Moncada, S., 1989. Role of endothelium-derived nitric oxide in the regulation of blood pressure. Proceeding of the National Academy of Science (USA) 86, 33753378.

Silva, F.A., Muccillo Baisch, A.L., Almeida, T.U., Castro, J.A., 1984. A vasodilator action of nicotine on the isolated superior mesenteric artery. In: Proceedings II Congress of the Brasilian Society of Pharmacology and Experimental Therapeutics. Brazilian Journal of Medical and Biological Research 17, 510.

Winquist, R.J., Bunting, P.B., Baskin, E.P., Wallace, A.A., 1984. Decreased endothelium-dependent relaxation in New Zeland genetic hypertensive rats. Journal of Hypertension 2, 536-541. 